\title{
„Behandlung der Blasenexstrophie“ 3. Kongreß der Europäischen Gesellschaft für Kinderurologie E.S.P.U., Cambridge März 1992
}

\author{
D. Kröpfl \\ Urologische Klinik und Poliklinik im Universitätsklinikum Essen
}

Das dritte jährliche Zusammentreffen der europäischen Gesellschaft für Kinderurologie fand statt in einem der ältesten College von Cambridge, das im 15. Jahrhundert gegründete Queens College. Das Hauptthema war die Behandlung der Blasenexstrophie. Dabei muß vorausgesetzt werden, daß der schrittweise funktionelle Blasenverschluß mit späterer Rekonstruktion des äußeren Genitals bei den meisten in Cambridge vertretenen Autoren als die Methode der Wahl für die Versorgung der Blasenexstrophie gilt.

Hinter den sehr unterschiedlichen absoluten Zahlen bezüglich des Erfolges verbergen sich insbesondere unterschiedliche Kriterien, mit denen die Kontinenz beurteilt wird. So reicht die Skala des Erfolges von 2stündiger Kontinenz unter Zuhilfenahme der Blasenaugmentation, des artifiziellen Sphinkters und des intermittierenden Katheterismus, bis zu den Kindern, die über 4 Stunden Tag und Nacht kontinent sind und die Blase vollkommen normal entleeren.

Die erste Gruppe der Arbeiten befaßte sich mit den Ergebnissen des primären funktionellen Blasenverschlusses mit sekundärer Rekonstruktion des Blasenhalses und des Genitals. Der primäre Verschluß war bej Frau Baka-Jakubiak aus Warschau und Mollard aus Lyon bei mehr als 90\% der Patienten erfolgreich. Mollard erreichte mit seiner Modifikation der Young-Dees-Blasenhalsrekonstruktion bei 36 von 54 so behandelten Patienten eine über 4 Stunden andauernde komplette Kontinenz. Eine 2-3stündige Kontinenz konnte Baka Jakubia bei $62 \%$ der Patienten erreichen.

In den Händen von Jeffs aus Baltimore war der primäre Blasenverschluß bei $97 \%$ der Kinder erfolgreich. Nach Erreichen der adäquaten Blasenkapazität von 60 bis $80 \mathrm{ml}$ führt er die Blasenhalsplastik und eine Antirefluxplastik durch. Eine komplette Kontinenz konnte bei $48 \%$ der Patienten und eine Kontinenz bis 3 Stunden bei weiteren $39 \%$ der Patienten erreicht werden. Das Erreichen der kompletten Kontinenz war altersabhängig. So

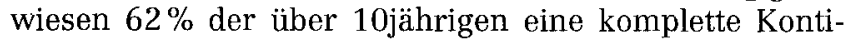
nenz auf. Im Gegensatz dazu war die komplette Kontinenz

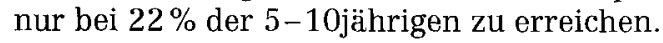

Im Gegensatz zu vorausgegangenen Ergebnissen waren die von Ransley aus dem Hospital for

Akt. Urol. 24 (1993) 106-109

(c) Georg Thieme Verlag Stuttgart · New York sick children in London präsentierten Daten, bezogen auf das Erreichen einer kompletten Kontinenz, wesentlich schlechter. Nach alleiniger Blasenhalsrekonstruktion haben nur 3 von 32 Patienten eine komplette Kontinenz erreicht. Aufgrund eines günstigen Verlaufes wird bei 6 weiteren Patienten die Kontinenz in der Zukunft erwartet und bei 4 Patienten wurde sie ermöglicht, entweder durch den intermittierenden Katheterismus oder den Einsatz des artifiziellen Sphinkters. Bei $68 \%$ wurde neben der Blasenhalsrekonstruktion entweder primär oder sekundär eine Blasenaugmentation durchgeführt, was zu einer Kontinenz bei $80 \%$ der so behandelten Patienten führte. Allerdings war bei allen Patienten der intermittierende Katheterismus notwendig. Darüber hinaus mußte $3 \times$ ein artifizieller Sphinkter und $5 \times$ die Technik nach Mitrofanoff zur Erreichung der Kontinenz angewandt werden.

Die Notwendigkeit einer Osteotomie wurde in Cambridge von den meisten Autoren als Voraussetzung für einen erfolgreichen Blasenverschluß und zur Erreichung der Kontinenz vorausgesetzt. Unter anderem wurde das begründet durch die schon 1989 von Husman veröffentlichen Daten, die zeigten, daß nur $60 \%$ der Kinder, die älter als 3 Tage und ohne eine Osteotomie verschlossen worden sind, die Kontinenz erreicht haben. Weniger einheitlich war die Wahl der Osteotomie. So wurden verschiedene Arbeiten über die am meisten verbreitete posteriore iliakale Osteotomie und über die in der neueren Zeit beschriebene anteriore iliakale und anteriore pelvine Osteotomie vorgestellt.

Perovic u. Mitarb. aus Belgrad präsentierten ihre große Erfahrung mit der von Frey und Cohen beschriebenen anterioren pelvinen Osteomie. Bei dieser Methode wird der Ramus superior ossis pubis möglichst medial des Ansatzes des Ligamentum inguinale durchgetrennt. Bei älteren Kindern wurde auch eine Fraktur des Ramus inferior ossis pubis bei einer Reaproximation der Symphyse von den Autoren in Kauf genommen und als belanglos beschrieben. Bei kurzer Beobachtungszeit von maximal 2,5 Jahren wiesen nur $5 \%$ eine Rediastase ohne weitere Folgen auf.

Bernard u. Mitarb. aus Frankreich wiesen auf ihre gute Erfahrung der posterioren iliakalen Osteotomie und gleichzeitiger Benutzung eines, für das Neugeborene und Kindesalter angepaßten Fixateur externe. Im weiteren Verlauf wiesen aber alle Kinder eine mit dem Wachstum zunehmende Rediastase der Symphyse auf. Khouri u. Mitarb. aus Paris beobachteten im Langzeit-Ver- 
lauf nach anteriorer iliakaler Osteotomie, die proximal des Akzeptabulums durchgeführt wird, das gleiche Problem. Alle 9 operierten Kinder entwickelten eine Rediastase der Symphyse. Als Vorteil der anterioren pelvinen und anterioren iliakalen Osteotomie kann das geringere Risiko des Auftretens von Beckenasymmetrien angenommen werden. Als sicherer Nachteil der anterioren iliakalen Osteotomie ist der relativ hohe Blutverlust und die von Jeffs beobachteten über 1 Jahr lang andauernden Paresen des Nervus femoralis. Die nächste Gruppe der Arbeiten befaßte sich mit der Behandlung des mißglückten Ekstrophieverschlusses und Reoperationen am Blasenhals.

Aufgrund der Erfahrung bei 29 Patienten berichtete Gearhart aus Baltimore über Prinzipien der Behandlung nach mißglücktem Exstrophieverschluß. So wies er auf die Notwendigkeit einer Osteotomie oder Reosteotomie hin; bei älteren Kindern sollte diese Maßnahme immer mit einem Fixateur externe kombiniert werden. Die Rekonstruktion des Blasenhalses sollte möglichst in der Kombination mit einer Augmentation der Blase mit detubularisiertem Darm erfolgen. Die Augmentation kann zum Erreichen der Kontinenz mit einem kontinenten Stoma im Sinne der Mitrofanoff-Operation oder mit einem artifiziellen Sphinkter kombiniert werden.

Die von Frau Fisch aus Mainz präsentierten Daten wiesen auf die Schwierigkeiten der erfolgreich wiederholten Rekonstruktion des Blasenhalses auch in der Kombination mit einer Blasenaugmentation hin. So konnte nur bei 1 von 8 so behandelten Patienten eine komplette Kontinenz erreicht werden. Weitere 5 Patienten wiesen eine entweder komplette oder partielle nächtliche Inkontinenz auf. Bei 3 Patienten war wegen einer kompletten Inkontinenz die Anlage eines Mainzpouches mit Appendixstoma notwendig. Die Kontinenz wurde erreicht bei 7 primär mit einem Kolon-Conduit versorgten Patienten mit einem Mainzpouch oder einer Sigmoideo-Sigmoidostomie. Im gleichen Zusammenhang wurde als weitere Entwicklung der in Mainz bevorzugten Uretero-Sigmoidostomie ein Sigma-Rektum-Pouch oder ein sog. Mainzpouch 2 vorgestellt. Bei diesem operativen Verfahren wird das orthotop gelegene Rektum mit einer aus der Kontinuität nicht ausgeschalteten Sigmaschlinge augmentiert. Als Ergebnis resultiert ein Darmreservoir ohne Hochdruckkontraktionen, so daß die Methode nach Angaben der Autoren auch für Patienten mit dilatierten Harnwegen geeignet ist.

Eine Reihe von Arbeiten befaßte sich mit der Blasensegmentation und Blasenersatz bei Kindern mit Blasenexstrophie. Hanna und Maggio aus New York berichteten über die Anwendung der intakten Zökum-Appendix-Einheit zur Rekonstruktion der Blase und Harnröhre bei einem Mädchen und drei Jungen. Die mögliche seminale Ausscheidung aus dem Ductus ejakulatorius wurde dabei nicht berücksichtigt. Indianapouch und verschiedene Dickdarmsegmente in der Kombination mit der Mitrofanoff-Technik wendete Bossiouny aus Kairo bei 15 Patienten mit Erfolg an. Alle Patienten mußten sich selbst katherisieren und bei 3 Patienten mußten größere Blasensteine offen chirurgisch entfernt werden. De Castro u. Mitarb. aus Bologna berichteten über eine normale oder erhaltene Nierenfunktion bei allen 15 mit Blasenaugmenta- tion behandelten Patienten. Darüber hinaus konnte bei 8 von 15 Patienten die angestrebte Kontinenz erreicht werden. Auch bei 4 von diesen Patienten wurden behandlungsbedürftige Blasensteine beobachtet.

In diesem Zusammenhang erscheint es mir sinnvoll, die im Vortrag von Ransley erwähnten und in der Arbeit von Holowell $\mathrm{u}$. Mitarb. im Bristish Journal of Urology 1991 veröffentlichten Daten über Komplikationen der Blasenaugmentation und des intermittierenden Katheterismus bei Exstrophiekindern zu erwähnen. Bei 6 Patienten perforierte die zu volle Blase beim von außen ausgeführten Druck und bei einem war die Perforation Folge des Katheterismus.

Besonders wichtig und betonenswert ist $\mathrm{zu}$ erwähnen die große Zahl der Eingriffe, die notwendig war, um den intermittierenden Katheterismus überhaupt zu ermöglichen. So mußte bei 3 Patienten die Harnröhre offen und bei 13 Patienten endoskopisch reoperiert werden.

Holowell u. Mitarb. aus London präsentierten urodynamische Daten über das Verhalten der Blase und der Harnröhre nach Augementation und Blasenhalsplastik. So wiesen alle 50 vorgestellten augmentierten Kinder eine Kapazität von mehr als $250 \mathrm{ml}$ auf. Bei 32 Kindern konnten keine Zeichen der Instabilität festgestellt werden. Bei 18 instabilen Blasen wiesen nur 5 Kinder Druckwellen von mehr als $35 \mathrm{~cm} \mathrm{H}_{2} \mathrm{O}$ auf. So konnte eine komplette Kontinenz unabhängig von dem Blasenverhalten bei allen Patienten mit einem mittleren Urethraverschlußdruck von $32 \mathrm{~cm} \mathrm{H}_{2} \mathrm{O}$ erreicht werden.

Über den Versuch, durch komplementäre Maßnahmen bei mißglückter Blasenhalsrekonstruktion die Kontinenz zu erreichen, berichtete Frau Röntgen aus Essen und stellte Ergebnisse der Implantation des artifiziellen Sphinkters vor. Die Kontinenz wurde erreicht bei 4 von 6 Patienten, von denen 5 zusätzlich augmentiert wurden. Diese relativ guten Ergebnisse wurden erkauft mit einer hohen Komplikationsrate, so daß bei diesen 6 Kindern insgesamt 10 Reoperationen notwendig waren.

Wichtig erscheint mir, auch 2 Arbeiten über die Histologie der exstrophierten Blasen zu erwähnen. So zeigten die elektronenmikroskopischen Untersuchungen von Szabo aus Ungarn, der 5 exstrophierte Blasen vor dem Verschluß untersuchte, ein für die normal angelegte und normal nervlich versorgte glatte Muskulatur typisches Bild. In diesem Zusammenhang soll man die letzten im British Journal of Urology 1991 veröffentlichten Daten von Holowell und Ransley erwähnen, die z.B. bei Kindern mit Epispadie vor der Blasenhalsrekonstruktion im Gegensatz zu dem postoperativen Befund normale urodynamische Befunde zeigen. Die Ergebnisse deuten auf die mögliche Störung der nervalen Versorgung der Blase im Rahmen der Blasenrekonstruktion. Schwöbel u. Mitarb. aus Zürich wiesen durch mikroskopische Untersuchungen von verschlossenen Blasenexstrophien bei 3 Kindern im Alter zwischen 4 und 9 Jahren eindeutige Komponenten der Dickdarmwand in der exstrophischen Blase. Als Erklärung postulierten die Autoren mögliche Übergangsformen zwischen den Cloacalen und Blasenexstro- 
phie. Sie stellen darüber hinaus die Frage, inwieweit Patienten mit einer Blasenexstrophie aufgrund der vorher beschriebenen Histologie dem Risiko der Entstehung eines Dickdarmkarzinoms ausgesetzt sind.

Die nächste Gruppe der Arbeiten beschäftigte sich mit Langzeitbeobachtungen der Blasenexstrophie-Patienten. Über die Langzeitergebnisse nach der Ureterosigmoideostomie bei Patienten mit Blasenexstrophie unter besonderer Berücksichtigung der sozialen Integration berichtete Prof. Hohenfellner aus Mainz. So überblickt die Mainzer Klinik 70 Patienten mit einer mittleren Beobachtungszeit von 15 Jahren und 20 Patienten mit einer mittleren Beobachtungszeit von 18 Jahren. In beiden Gruppen wiesen mehr als $90 \%$ der Patienten eine vor dem Schulalter erreichte komplette Kontinenz auf. Eine abgeschlossene schulische Ausbildung erreichten 38 von den 40 Patienten und mehr als $90 \%$ der über 21 jährigen hatten eine Beschäftigung, die eine berufliche Qualifikation abverlangt.

Über Langzeitergebnisse nach der Ureterosigmoido-Sigmoideostomie bei 38 Patienten berichteten Lottmann u. Mitarb. aus Paris. Tagsüber waren $81 \%$ und nachts über $74 \%$ der Operierten komplett kontinent. Bei diesem schrittweisen Vorgehen wurden die Kinder zuerst mittels eines Kolon-Conduits mit antirefluxiver Harnleiter-Neueinpflanzung abgeleitet und im späteren Verlauf erfolgte eine Rückverlagerung des Conduits in eine Sigmoideo-Sigmoideostomie. Bei der mittleren Beobachtungszeit von $10 \mathrm{Jahren}$ wiesen nur $2 \%$ benigne Dickdarmtumoren, aber sogar 21 von 38 Patienten rezidivierende Pyelonephritis-Attacken auf. Analog den Ergebnissen aus der Mainzer Klinik war die soziale Integration bei den meisten Patienten normal.

Der Frage der genitalen und sexuellen Rehabilitation bei Patienten mit Blasenexstrophie haben sich mehrere Autoren gewidmet. Mellin u. Mitarb. aus Paris untersuchten dabei 80 Männer und 38 Frauen, älter als 18, die in ihrem Krankengut von 184 Patienten langzeitig beobachtet wurden. So wurden von 38 Frauen 9 primär rekonstruiert und 29 abgeleitet. Einen Gebärmutterprolaps, eine für diese Gruppe der Patienten typische Komplikation, wiesen 7 Patientinnen auf. 32 plastisch-chirurgische Eingriffe waren notwendig, um bei den Patientinnen einen gewünschten kosmetischen Aspekt bzw. einen normalen Geschlechtsverkehr zu ermöglichen. 14 Frauen wurden $16 \times$ schwanger, wobei alle Schwangerschaften mittels eines Kaiserschnittes beendet wurden. Im Gegensatz zu Frauen wurde ein zufriedenstellender Geschlechtsverkehr nur bei 36 bzw. $50 \%$ der Männer beobachtet. 50 Patienten berichteten über Ejakulationen. Interessanterweise waren 14 von 15 Patienten, die insgesamt 23 Kinder gezeugt haben, primär abgeleitet und nicht einer Rekonstruktion des Blasenhalses unterzogen. Die soziale Integration des beschriebenen Kollektivs wurde von den Untersuchern bei den meisten als normal bezeichnet.

Jaureguizar u. Mitarb. aus Madrid untersuchten den genitalen Aspekt der Exstrophie bei den in den letzten 20 Jahren in deren Klinik operierten Patienten. Bei 48 Männern, die einer Rekonstruktion des Geni- tales unterzogen wurden, konnte eine mittlere Verlängerung des Gliedes von ca. $2 \mathrm{~cm}$ erreicht werden. Eine normale Erektion ohne Deviation wiesen 28 und mit Deviation 19 Patienten auf.

Von 23 behandelten Patientinnen war bei 17 eine Rekonstruktion der suprapubischen Region, und bei 8 eine vaginale Plastik notwendig.

In diesem Zusammenhang möchte ich noch auf eine sehr interessante Arbeit über die plastische Rekonstruktion des Mons pubis und der Bauchwand bei Frauen berichten. De Castro u. Mitarb. aus Bologna haben zu diesem Zwecke in der 1. Sitzung in Allgemeinnarkose bilateral der typischen Mediannarbe subkutan je einen $500 \mathrm{~cm}^{3}$ großen Hautexpander eingebaut. Die Expander wurden wöchentlich gefüllt und erreichten nach 6 Wochen ein Volumen von ca. $800 \mathrm{~cm}^{3}$. Das ermöglichte bilateral das Gewinnen von ausreichendem Gewebe zur Bildung von vitalen Hautfettschwenklappen, die dann zur Rekonstruktion der Narbe und Hautdeckung benutzt wurden.

\section{Zusammenfassung}

Nach Meinung der meisten in Cambridge vertretenen Autoren ist die Behandlung der Wahl bei der Blasenexstrophie der primäre Verschluß mit sekundärer Rekonstruktion des Blasenhalses und des Genitals. Eine Osteotomie ist bei allen Kindern, älter als 72 Std., obligat. In erfahrenen Händen ist der primäre Verschluß in der Regel erfolgreich, so daß sich Mißerfolge hauptsächlich auf die Kontinenz beziehen. Ist der Blasenverschluß erfolgreich, so ist bei ca. $5 \%$ der kontinenten Patienten mit einer Verschlechterung der Nierenfunktion zu rechnen. Eine neurogene Schädigung der verschlossenen Blase ist wahrscheinlich sehr häufig. Bei vielen Kindern wird der Weg bis zu einer Kontinenz durch wiederholte Eingriffe begleitet. Auch in den besten Händen wird die erhoffte Kontinenz häufig erst nach der Pubertät erreicht.

Welche Alternativen haben wir zum primären Blasenverschluß? Eine kontinente Harnableitung, wie Ureterosigmoideostomie, ohne Urostoma ist in vielerlei Hinsicht eine verlockende Maßnahme. Die Kontinenz wird sehr früh erreicht und die Kinder können in einer wichtigen Entwicklungsphase, falls keine Komplikationen auftreten, fast arztfrei leben. Leider ist die Inzidenz der Spätkomplikationen nach der Ureterosigmoideostomie wie Pyelonephritis und Steinbildung recht häufig, mit einer Einschränkung der Nierenfunktion ist bei mindestens $10 \%$ dieser Kinder zu rechnen. Als besonders gravierend erscheint mir aber die Induktion der Dickdarmkarzinome, die bei ca.10\% der Patienten mit Ureterosigmoideostomie zu erwarten ist.

Faßt man das zusammen, erscheint der primäre Blasenverschluß mit späterer Rekonstruktion des Blasenhalses und des Genitals als die Methode der Wahl zur Behandlung der Blasenexstrophie. Ein einzeitiger Verschluß der Blase mit Blasenrekonstruktion und Rekonstruktion des Genitals wie von Schrott propagiert wird, muß dabei als Alternative erwähnt werden. 
Wird die erhoffte Kontinenz durch die 1. Rekonstruktion des Blasenhalses nicht erreicht, so ist bei einem solchen Kind eine Ureterosigmoideostomie oder eine kontinente Harnableitung mit Hautstoma wiederholten frustranen Reoperationen oder sogar dem Einbau einem artifiziellen Sphinkter mit konsekutiven Komplikationen vorzuziehen.
Dr. Kröpfl

Urologische Klinik und Poliklinik Universitätsklinikum

Hufelandstr. 55

4300 Essen 